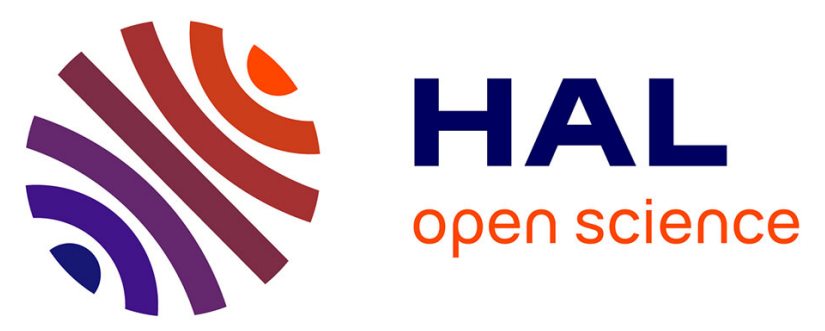

\title{
HYR2PICS: Hybrid Regularized Reconstruction for combined Parallel Imaging and Compressive Sensing in MRI
}

Claire Boyer, Philippe Ciuciu, Pierre Weiss, Sébastien Meriaux

\section{> To cite this version:}

Claire Boyer, Philippe Ciuciu, Pierre Weiss, Sébastien Meriaux. HYR2PICS: Hybrid Regularized Reconstruction for combined Parallel Imaging and Compressive Sensing in MRI. IEEE International Symposium on Biomedical Imaging, May 2012, Barcelone, Spain. cea-00691623

HAL Id: cea-00691623

https://hal-cea.archives-ouvertes.fr/cea-00691623

Submitted on 26 Apr 2012

HAL is a multi-disciplinary open access archive for the deposit and dissemination of scientific research documents, whether they are published or not. The documents may come from teaching and research institutions in France or abroad, or from public or private research centers.
L'archive ouverte pluridisciplinaire HAL, est destinée au dépôt et à la diffusion de documents scientifiques de niveau recherche, publiés ou non, émanant des établissements d'enseignement et de recherche français ou étrangers, des laboratoires publics ou privés. 


\title{
HYR²PICS: HYBRID REGULARIZED RECONSTRUCTION FOR COMBINED PARALLEL IMAGING AND COMPRESSIVE SENSING IN MRI
}

\author{
Claire Boyer $^{(1,2)}$, Philippe Ciuciu ${ }^{(1)}$, Pierre Weiss ${ }^{(2)}$ and Sébastien Mériaux ${ }^{(1)}$ \\ ${ }^{(1)}$ NeuroSpin, CEA Saclay, Bât. 145, 91191 Gif-sur-Yvette, cedex France \\ (2) INSA Toulouse (Maths. Dpt) and IMT (UMR CNRS 5219), Toulouse, France. \\ 1 philippe.ciuciu, sebastien.meriaux\}@cea.fr, ${ }^{2}$ \{claire.boyer11, pierre.armand.weiss\}@gmail.com
}

\begin{abstract}
Both parallel Magnetic Resonance Imaging (pMRI) and Compressed Sensing (CS) are emerging techniques to accelerate conventional MRI by reducing the number of acquired data in the $k$-space. So far, first attempts to combine sensitivity encoding (SENSE) imaging in pMRI with CS have been proposed in the context of Cartesian trajectories. Here, we extend these approaches to non-Cartesian trajectories by jointly formulating the CS and SENSE recovery in a hybrid Fourier/wavelet framework and optimizing a convex but nonsmooth criterion. On anatomical MRI data, we show that $\mathrm{HYR}^{2} \mathrm{PICS}$ outperforms wavelet-based regularized SENSE reconstruction. Our results are also in agreement with the Transform Point Spread Function (TPSF) criterion that measures the degree of incoherence of $k$ space undersampling schemes.
\end{abstract}

Index Terms - MRI, parallel imaging, Compressive sensing, wavelets, nonsmooth optimization.

\section{INTRODUCTION}

Reducing scanning time in MRI exams remains a worldwide challenging issue. The expected benefits of a faster acquisition can be summarized as follows: $i$.) limit patient's exposure to the MRI environment for safety reasons, ii.) maintain a strong robustness in the acquisition with respect to subject's motion, iii.) limit geometric distortions. The basic idea to make MRI acquisitions faster consists of reducing the amount of acquired samples in the $k$-space and to develop dedicated reconstruction pipelines. To this end, both parallel imaging (pMRI) and compressed sensing (CS) have been proposed. pMRI relies on a geometrical principle involving multiple receiver coils with complementary sensitivity profiles that makes the reconstruction of MR images from multichannel $k$-space data sampled below the Nyquist sampling rate feasible. Standard reconstruction methods include sensitivity-based single image approaches like SENSE [1], SMASH [2] and autocalibrating coil-by-coil techniques like GRAPPA [3] and the most recent SPIRiT algorithm [4], which draws its inspiration from both GRAPPA and SENSE. Theoretically, the acceleration factor $R$ is upper bounded by the number of channels under ideal conditions. In practice this bound is never achieved due the amount of noise and imperfect coil geometry.

CS-MRI relies on the Compressed Sensing theory [5,6] that proposes a new framework for data sampling and signal recovery. It has generated significant interest because it enables exact signal reconstruction from much fewer data samples than suggested by Shannon's theorem. CS-MRI has been popularized because MR images meet the two key assumptions underlying the CS reconstruction: $a$.) MRI images are sparse in a given linear transform domain and b.) the Fourier encoding is incoherent with this sparse transformation.
CS-MRI methods include SparseMRI for Cartesian trajectories [7] and other approaches for alternative sampling schemes [8].

The idea of combining CS and pMRI has been proposed recently in an attempt of further accelerating SENSE imaging because pMRI and CS-MRI reduce sampling according to different ancillary information (channel sensitivity vs. image sparsity). The SparseSENSE method and its equivalence [9] have been developed as a straightforward combination method that reconstructs images from multichannel data using the nonlinear SparseMRI solver, except that Fourier encoding is replaced by the sensitivity encoding comprising Fourier encoding and sensitivity weighting. In [10], a more efficient method with guaranteed incoherence, called CS-SENSE, was proposed for Cartesian trajectories only. Its principle is based on a two-step procedure, where CS reconstruction is first carried out by SparseMRI for the aliased image of each channel and second, Cartesian SENSE reconstruction is performed in the image domain. Here, we extend the CS-SENSE approach to non Cartesian trajectories and in this respect our algorithm is closer to SPIRiT. Our method named HYR ${ }^{2}$ PICS is hybrid in several respects: i.) it jointly formulates CS and pMRI in the Fourier space (see Section 2) so as to address the reconstruction of full Field Of View (FOV) images in the wavelet domain from uncoherent multichannel Fourier samples. ii.) It combines regularization in the wavelet and image domains so as to promote sparsity while avoiding ringing artefacts. iii.) It makes use of recent optimal primal-dual convex but non-smooth minimization algorithm (see Section 3). In section 4, the TPSF criterion expressed in the pMRI context demonstrates the gain induced by incoherent sampling. Section 5 is devoted to validation on anatomical data, where it is shown that the HYR ${ }^{2}$ PICS method outperforms wavelet-based regularized SENSE reconstruction: the most accurate results are obtained for 2D random point or checkerboard sampling schemes, thus departing from conventional pMRI undersampling.

\section{PROBLEM STATEMENT}

In this work, we consider discrete complex spin densities $\rho \in \mathbb{C}^{n}$, where $n=n_{x} \cdot n_{y}$ is the pixels number. $\rho(x)$ denotes the value of $\rho$ at pixel $x \in\{1, \cdots, n\}$. All the theory developed here also applies to $3 \mathrm{D}$ densities, and then $n=n_{x} \cdot n_{y} \cdot n_{z}$. The standard inner product is denoted $\langle\cdot, \cdot\rangle$. The associated norm is denoted $\|\cdot\|$.

The canonical basis of $\mathbb{R}^{n}$ is composed of the vectors $e_{1}, \cdots, e_{n}$. Let $\boldsymbol{A}$ and $\boldsymbol{B}$ be two matrices $[\boldsymbol{A}, \boldsymbol{B}]$ and $[\boldsymbol{A} ; \boldsymbol{B}]$ respectively denote a horizontal and a vertical concatenation of $\boldsymbol{A}$ and $\boldsymbol{B}$ (as in Matlab). $\mathbf{I d}_{n}=\left[e_{1}, \ldots, e_{n}\right]$ denotes the identity operator in $\mathbb{R}^{n}$ or $\mathbb{C}^{n}$. Let $\boldsymbol{A}$ be a linear application. $\boldsymbol{A}^{*}$ denotes its adjoint (conjugate transpose). $\boldsymbol{F}$ denotes the discrete Fourier Transform. It is a unitary transform so that $\boldsymbol{F}^{*}=\boldsymbol{F}^{-1}$. $\boldsymbol{\partial}_{1}: \mathbb{C}^{n} \rightarrow \mathbb{C}^{n}$ and $\boldsymbol{\partial}_{2}: \mathbb{C}^{n} \rightarrow \mathbb{C}^{n}$ are linear applications that design the discretized derivatives in the $x, y$ 
directions respectively. $\boldsymbol{\nabla}=\left[\boldsymbol{\partial}_{1} ; \boldsymbol{\partial}_{2}\right]$ denotes the discrete gradient.

Let $\mathcal{F}: \mathbb{R}^{n} \rightarrow \mathbb{R} \cup\{+\infty\}$ be a convex, closed function with non-empty domain. $\mathcal{F}^{\dagger}$ refers to the Fenchel conjugate of $\mathcal{F}$ defined by: $\mathcal{F}^{\dagger}(u)=\sup _{v \in \mathbb{R}^{n}}\langle u, v\rangle-\mathcal{F}(v)$. The sub-differential of $\mathcal{F}$ at $u$ is the set defined by: $\boldsymbol{\partial \mathcal { F }}(u)=\left\{\eta \in \mathbb{R}^{n}, \mathcal{F}(v) \geq \mathcal{F}(u)+\langle\eta, v-\right.$ $\left.u\rangle, \forall v \in \mathbb{R}^{n}\right\}$. The resolvent (or proximal operator) of $\mathcal{F}$ at point $u$ is defined by: $(\mathbf{I d}+\boldsymbol{\partial} \mathcal{F})^{-1}(u)=\arg \min _{v \in \mathbb{R}^{n}} \mathcal{F}(v)+\|v-u\|^{2} / 2$.

\subsection{Parallel imaging}

In parallel MRI, an array of $L$ coils is employed to measure the spin density $\rho$ into the object under investigation. The signal $d_{\ell} \in \mathbb{C}^{m}$ received by each coil $\ell(1 \leq \ell \leq L)$ is the Fourier transform of the desired 2D field $\rho$ on the specified FOV weighted by the coil sensitivity profile, evaluated at some location. This signal is deteriorated by an additive Gaussian white noise $n_{\ell}$ of variance $\sigma_{\ell}$.

In this work, we consider the problem in the discrete setting. We assume that the spin densities $\rho$ are defined on a Euclidean grid which is a discretization of the FOV. Under this assumption, the acquisition process can be written in the condensed form:

$$
d_{\ell}=\boldsymbol{\Sigma}_{s} \boldsymbol{F} \boldsymbol{S}_{\ell} \rho+n_{\ell}
$$

where $\boldsymbol{S}_{\ell}: \mathbb{C}^{n} \rightarrow \mathbb{C}^{n}$ denotes a sensitivity operator. It is a diagonal matrix the $i$-th diagonal element of which indicates the attenuation at pixel $i . \boldsymbol{\Sigma}_{s}: \mathbb{C}^{n} \rightarrow \mathbb{C}^{m}$ represents the sampling operator. This matrix reads $\boldsymbol{\Sigma}_{s}=\left[e_{i_{1}}, \cdots, e_{i_{m}}\right]^{*}$ where each $i_{j}$ indicates the index of the $j$-th measure on the Cartesian grid. Eq. (1) presents the advantage to unify pMRI and compressive sampling by considering different structures for matrix $\boldsymbol{\Sigma}_{s}: i$.) A full k-space sampling would correspond to $\boldsymbol{\Sigma}_{s}=\mathbf{I d}_{n}$. ii.) Compressive sensing would lead to a random choice of $m$ indices $\left\{i_{1}, \cdots, i_{m}\right\}$ in the set $\{1, \cdots, n\}$.

\subsection{Combining CS and pMRI reconstructions}

Since the between-channel covariance is assumed diagonal $\boldsymbol{\Lambda}=$ $\operatorname{diag}\left[\sigma_{1}^{2} \mathbf{I d}, \cdots, \sigma_{L}^{2} \mathbf{I d}\right]$, considering all incomplete data $\left(d_{\ell}\right)_{\ell=1: L}$, the SENSE solution amounts to minimizing the following criterion:

$$
\mathcal{H}(\rho)=\sum_{\ell=1}^{L} \sigma_{\ell}^{-1}\left\|d_{\ell}-\boldsymbol{\Sigma}_{s} \boldsymbol{F} \boldsymbol{S}_{\ell} \rho\right\|^{2} .
$$

This minimization boils down to the resolution of a linear system which can be solved in $O(n \log (n))$ operations using Fourier transforms. However, the operator $\boldsymbol{\Sigma}_{s} \boldsymbol{F} \boldsymbol{S}_{\ell}$ is usually rank defficient or has small singular values since the sensitivities have a fast spatial decay. Minimizing (2) thus yields unstable results.

An appropriate remedy consists of regularizing the problem by imposing sparsity constraints. As pointed out in [7], MR images are usually sparse in certain transform domains, such as wavelets representations. Let $\Psi_{i} \in \mathbb{C}^{n}$ design an atom and $\boldsymbol{\Psi}=\left[\Psi_{1}, \ldots, \Psi_{p}\right] \in$ $\mathbb{C}^{n \times p}, p \geq n$ design a dictionary. In the following, we assume that $\boldsymbol{\Psi}$ is surjective. A spin density $\rho$ is said to be sparse in $\boldsymbol{\Psi}$ if it can be written $\rho=\sum_{i=1}^{p} \zeta(i) \Psi_{i}=\boldsymbol{\Psi} \zeta$ where $\zeta \in \mathbb{C}^{n}$ has only a few non-zero entries. In the following, we employ a dyadic 2D orthonormal wavelet transformation over $j_{\max }$ resolution levels.

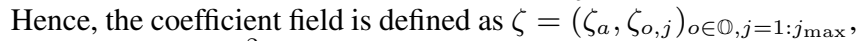
where $\mathbb{O}=\{0,1\}^{2} \backslash\{(0,0)\} \zeta_{a}=\left(\zeta_{a}, k\right)$ represents the vector of approximation coefficients and $\zeta_{o, j}=\left(\zeta_{o, j, k}\right)_{k=1: K_{j}}$ denotes the vector of detail coefficients at resolution level $j$ and orientation $o$, with $K_{j}=n 2^{-2 j}$. Note that in the dyadic case, there are three orientations corresponding to the horizontal $(h)$, vertical $(v)$, and diagonal $(d)$ directions.
The compressive sensing theory ensures that $\rho$ can be recovered precisely with only few observations $d(k)$, with $k \in\{1, \ldots, m\}$ and $m \ll n$, by computing $\widehat{\rho}=\mathbf{\Psi} \widehat{\zeta}$ where

$$
\widehat{\zeta} \in \underset{\zeta \in \mathbb{C}^{p}}{\arg \min }\|\zeta\|_{1} \quad \text { s.t. } \quad d(k)=\left\langle\rho, \phi_{k}\right\rangle, \forall k=1: m .
$$

In SparseMRI [7] where single coil acquisition is addressed, the acquisition basis $\boldsymbol{\Phi}=\left[\phi_{1}, \ldots, \phi_{n}\right]$ identifies with $\boldsymbol{F}$. In more recent contributions [10] where parallel imaging is involved, the basis becomes channel-specific i.e., $\boldsymbol{\Phi}_{\ell}=\boldsymbol{F} \boldsymbol{S}_{\ell}$. In the presence of noise, the constraint $d(k)=\left\langle\rho, \phi_{k}\right\rangle$ is relaxed. Moreover, regularizations based solely on sparsity (synthesis) lead to spurious oscillations that can be avoided using regularization in the spatial domain (analysis). Hence, following [11], we look for the decomposition $\zeta$ of $\rho$ in the dictionary $\Psi$ by minimizing the following criterion that embodies hybrid regularization (in the wavelet and image domains):

$$
\widehat{\zeta} \in \underset{\zeta \in \mathbb{C}^{n}}{\arg \min }\left[\mathcal{J}_{\mathrm{WLS}}(\boldsymbol{\Phi} \boldsymbol{\Psi} \zeta)+\mathcal{J}_{S}(\zeta)+\lambda_{A} \mathcal{J}_{A}(\boldsymbol{\nabla} \boldsymbol{\Psi} \zeta)\right] .
$$

We then reconstruct the image solution as $\widehat{\rho}=\boldsymbol{\Psi} \widehat{\zeta}$. In Eq. (4), $\boldsymbol{\Phi}=\left[\boldsymbol{\Sigma}_{s} \boldsymbol{F} \boldsymbol{S}_{1} ; \ldots ; \boldsymbol{\Sigma}_{s} \boldsymbol{F} \boldsymbol{S}_{L}\right]$ is the observation operator.

$\mathcal{J}_{\mathrm{WLS}}(\boldsymbol{\Phi} \rho)=\sum_{\ell=1}^{L} \sigma_{\ell}^{-1}\left\|d_{\ell}-(\boldsymbol{\Phi} \rho)_{\ell}\right\|^{2} . \mathcal{J}_{S}$ is the penalization in the wavelet domain that ensures sparsity of $\zeta$ :

$$
\mathcal{J}_{S}(\zeta)=\sum_{k=1}^{K_{j_{\max }}} \varphi_{a}\left(\zeta_{a, k}\right)+\sum_{o \in \mathbb{O}} \sum_{j=1}^{j_{\max }} \sum_{k=1}^{K_{j}} \varphi_{o, j}\left(\zeta_{o, j, k}\right),
$$

where we have $\forall o \in \mathbb{O}$ and $j \in\left\{1, \ldots, j_{\max }\right\}, \varphi_{o, j}(\xi)=\varphi_{o, j}^{\operatorname{Re}}(\xi)+$ $\varphi_{o, j}^{\operatorname{Im}}(\xi) \forall \xi \in \mathbb{C}$, with $\varphi_{o, j}^{\diamond}(\xi)=\alpha_{o, j}^{\diamond}\left|\diamond\left(\xi-\mu_{o, j}\right)\right|+\beta_{o, j}^{\diamond} \mid \diamond(\xi-$ $\left.\mu_{o, j}\right)\left.\right|^{2}$ and $(\diamond=\operatorname{Re})$ or $(\diamond=\operatorname{Im}), \mu_{o, j}=\mu_{o, j}^{\mathrm{Re}}+\imath \mu_{o, j}^{\operatorname{Im}} \in \mathbb{C}$ and $\alpha_{o, j}^{\diamond}, \beta_{o, j}^{\diamond}$ are some positive real constants. Hereabove, $\operatorname{Re}(\cdot)$ and $\operatorname{Im}(\cdot)$ (or ${ }^{\operatorname{Re}}$ and $\cdot{ }^{\operatorname{Im}}$ ) stand for the real and imaginary parts, respectively. A quadratic penalty term $\varphi_{a}$ is adopted for $\zeta_{a}$.

In eq. (4), $\mathcal{J}_{A}(\nabla \rho)=\sum_{i=1}^{n} \varphi_{A}\left(\sqrt{\left(\boldsymbol{\partial}_{1} \rho\right)(i)^{2}+\left(\boldsymbol{\partial}_{2} \rho\right)(i)^{2}}\right)$ where $\varphi_{A}$ is a Huber function and $\lambda_{A}$ a non-negative regularization parameter. $\mathcal{J}_{A}(\nabla \rho)$ is thus a smooth approximation of the total variation, allowing to avoid staircase effect. As regards hyper-parameter estimation, we used a Maximum Likelihood (ML) estimation strategy on a reference image, typically the SENSE reconstruction, that provides fair estimates for $\lambda_{A}$ and $\left(\boldsymbol{\alpha}^{\diamond}, \boldsymbol{\beta}^{\diamond}\right)$ in a reasonable computation time. As proposed in [12], more accurate but more costly estimates can be achieved using stochastic sampling.

\section{PRIMAL-DUAL OPTIMIZATION}

\subsection{A review of optimization methods}

Minimizing criterion (4) is a hard task since the problem is convex, but large scale, non differentiable and non strongly convex. Many first order schemes were recently proposed or rediscovered by the signal processing community to solve such problems.

Among them, let us cite proximal gradient descents (also called Forward-Backward splitting) and their acceleration [13], DouglasRachford algorithms, ADMM and their extension to more components such as Parallel ProXimal Algorithm (PPXA) [14], or extragradient Like methods [15]. All these methods rely on the ability to compute resolvents of a part of the problem explicitely. Among the above methods it seems that only Extra-gradient like methods can be applied directly to problem (2). Indeed :

- Proximal gradient descents would require the computation of the resolvents of $\mathcal{J}_{A}(\rho)$ which cannot be done explicitely.

- ADM or Douglas-Rachford like methods rely on the ability to solve linear systems of kind $\left(\boldsymbol{S}_{\ell}^{*} \boldsymbol{F}^{*} \boldsymbol{\Sigma}_{s}^{*} \boldsymbol{\Sigma}_{s} \boldsymbol{F} \boldsymbol{S}_{\ell}+\mathbf{I d}\right) x=b$, 
which is an open topic in linear algebra.

We thus decided to use extra-gradient like methods and more particularly the Chambolle-Pock (CP) primal-dual method, which has an "optimal" $O\left(\frac{1}{k}\right)$ convergence rate for the problem under consideration and only requires matrix-vector multiplications. In contrast to most contributions (e.g. [16]), this scheme is not composed of two nested iterative algorithms. Such methods usually have no convergence proof due to the approximations made in the inner iteration. Note however that a few primal-dual alternative algorithms have been proposed in the recent literature (see [17] and references therein) that could possibly perform the same optimization task.

\subsection{The Chambolle-Pock implementation}

Problem (4) can be rewritten as:

$$
\min _{x \in \mathbb{C}^{p}} \mathcal{F}(\boldsymbol{A} x)+\mathcal{G}(x) .
$$

where $\mathcal{F}(y)=\mathcal{J}_{\mathrm{WLS}}\left(y_{1}\right)+\lambda_{A} \mathcal{J}_{A}\left(y_{2}\right)$ with $y=\left[y_{1} ; y_{2}\right] \in \mathbb{C}^{m L} \times$ $\mathbb{C}^{2 n}, \mathcal{G}(x)=\mathcal{J}_{S}(x)$ and $\boldsymbol{A}=[\boldsymbol{\Phi} \boldsymbol{\Psi} ; \boldsymbol{\nabla} \boldsymbol{\Psi}]$. This formulation has the nice property that both $\mathcal{G}$ and $\mathcal{F}$ are separable pixel-wise, making their resolvent computation very easy. The primal-dual optimality conditions of this problem write $\boldsymbol{A} \hat{x} \in \boldsymbol{\partial} \mathcal{F}^{\dagger}(\hat{y})$ and $-\left(\boldsymbol{A}^{*} \hat{y}\right) \in$ $\partial \mathcal{G}(\hat{x})$. These conditions amount to say that $(\hat{x}, \hat{y})$ is a saddle-point. Such equilibrium can be reached by iterating primal descents and dual ascents sequentially. The CP implementation of this idea writes:

$$
\left\{\begin{aligned}
y_{k+1} & =\overbrace{\left(\mathbf{I d}+\sigma \boldsymbol{\partial} \mathcal{F}^{\dagger}\right)^{-1}}^{\text {prox }_{\sigma \mathcal{F}^{\dagger}}}\left(y_{k}+\sigma \boldsymbol{A} \bar{x}_{k+1}\right) \\
x_{k+1} & =\underbrace{(\mathbf{I d}+\tau \boldsymbol{\partial})^{-1}}_{=\operatorname{prox}_{\tau \mathcal{G}}}\left(x_{k}-\tau \boldsymbol{A}^{*} y_{k+1}\right) \\
\bar{x}_{k+1} & =2 x^{k+1}-x^{k}
\end{aligned}\right.
$$

where the last step is a correction term (extra-gradient) and the parameters $\sigma$ and $\tau$ should satisfy $\sigma \tau=L^{2}$ where $L$ is the highest singular value of $\boldsymbol{A}$. In practice, this algorithm requires less than 50 iterations to give a solution precise enough for the visual system.

\section{OPTIMIZING THE UNDERSAMPLING SCHEME}

Once the acceleration factor $R$ has been chosen, the remaining degrees of freedom lie in the $k$-space samples we really pick up for data acquisition. In the pMRI context, the undersampling scheme is fixed and consists of choosing one out of $R$ phase encoding lines as illustrated in Fig. 1(a). In order to consider pseudo-random schemes (see Fig. 1(b)-(f)) while allowing exact reconstruction in the CS context, the concept of incoherence has been introduced in $[5,6]$.
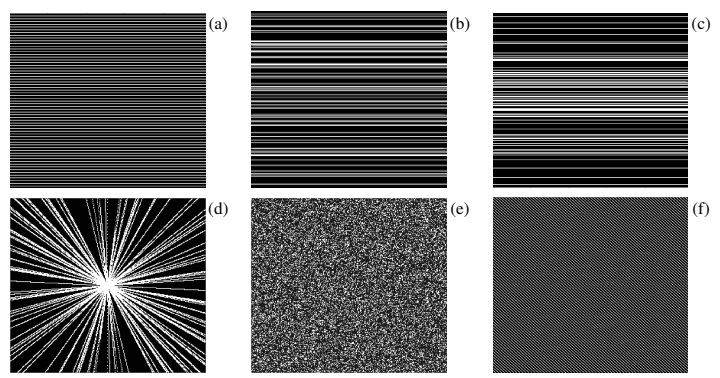

Fig. 1. Various $k$-space sampling schemes for $R=4$ : selected points appear in white color. (a) pMRI line undersampling. (b)(c): Pseudo-random line undersampling with uniform (b) and Gaussian (c) distributions. (d): Radial scheme with uniformly random angles. (e): 2D random points. (f): checkerboard scheme.

\subsection{Incoherent sampling}

Incoherence extends the duality between time and frequency and expresses the idea that objects with a sparse representation must be spread out in the domain in which they are acquired. There exists different measures of coherence including restricted isometry properties [5] or mutual incoherence [6]. In this work, we use mutual incoherence first introduced in [7].

The coherence between the sensing basis $\boldsymbol{\Phi}$ and the representation basis $\boldsymbol{\Psi}$ can be measured by $\gamma(\boldsymbol{\Psi}, \boldsymbol{\Phi})=\max _{1 \leq k, j \leq n} \frac{\left|\left\langle\Phi_{k}, \Psi_{j}\right\rangle\right|}{\left\|\Phi_{k}\right\| \cdot\left\|\Psi_{j}\right\|}$, where $\boldsymbol{\Phi}=\left[\Phi_{1}, \ldots, \Phi_{m L}\right]^{*}$. In other words, coherence measures the largest correlation between the lines of $\boldsymbol{\Psi}$ and columns of $\boldsymbol{\Phi}$. This notion of coherence can also be captured by a variant called mutual coherence that reads:

$$
\mu=\mu(\boldsymbol{\Phi} \boldsymbol{\Psi})=\max _{1 \leq i, j \leq p, i \neq j} \frac{\left|\left\langle\boldsymbol{\Phi} \boldsymbol{\Psi} e_{i}, \boldsymbol{\Phi} \boldsymbol{\Psi} e_{j}\right\rangle\right|}{\left\|\boldsymbol{\Phi} \boldsymbol{\Psi} e_{i}\right\| \cdot\left\|\boldsymbol{\Phi} \boldsymbol{\Psi} e_{j}\right\|}
$$

One of the typical results relating coherence to sparsity states that if $\rho=\Psi \zeta$ is $s$-sparse and $s \leq \frac{1+1 / \mu}{2}$ then the exact recovery of $\rho$ can be achieved by solving Eq. (3). As a consequence, the smaller the coherence, the fewer samples are needed. To reduce the number of MRI samples, we can either play with $\boldsymbol{\Psi}$ so as to decrease $S$ or minimize the mutual coherence $\mu$ by varying the sampling scheme.

\subsection{Coherence in parallel imaging}

In this work, we distinguished different undersampling schemes like those appearing in Fig. 1. As shown in Tab. 1, the trajectory that presents the best coherence (lowest $\mu$ ) is the random-points pattern irrespective of $R$, while the worst one remains the Normally Distributed (ND) random rows pattern. This is quite surprising since the $k$-space center contains most of the signal energy. As the random point scheme is not physically feasible, we will select the closest one i.e., the checkerboard downsampling, which consists of picking one pixel over $R$ per row, and shifting rows of one pixel one to another. Results for radial schemes seem conflicting: for $R=2$, the Uniformly Distributed (UD) angled scheme brings a lower $\mu$ value than the equi-angled one, while the converse holds for $R=4$. Finally, whatever the scheme we look at in Tab. 1, the mutual coherence criterion increases with $R$ so that incoherence decreases illustratring the negative impact of increasing $R$ while keeping the sampling scheme in a $2 \mathrm{D}$ setting (see [7] for more general 3D schemes).

Table 1. Mutual coherence $\mu$ in pMRI for $R=2$ and $R=4$.

\begin{tabular}{c||c|c}
\hline Undersampling scheme & $\mu(R=2)$ & $\mu(R=4)$. \\
\hline \hline Regular lines & 0.2770 & 0.6774 \\
\hline ND lines & 0.4850 & 0.7471 \\
\hline UD lines & 0.3336 & 0.4602 \\
\hline Random points & 0.1040 & 0.1739 \\
\hline Checkerboard & 02373 & 0.4020 \\
\hline UD angled radial & 0.2526 & 0.6233 \\
\hline Equi-angled radial & 0.2637 & 0.4234 \\
\hline Spiral scheme & 0.3214 & 0.3499 \\
\hline
\end{tabular}

\section{RESULTS}

\subsection{Experimental data}

For validation purpose, we acquired anatomical MRI data on a 3T Siemens Trio magnet using a $L=32$ channel head coil and parallel 
imaging. We used a 3D T1-weighted MP-RAGE pulse sequence at a $1 \times 1 \times 1.1 \mathrm{~mm}^{3}$ spatial resolution $(T E=2.98 \mathrm{~ms}, T R=2300 \mathrm{~ms}$, slice thickness $=1.1 \mathrm{~mm}$, FOV $=256 \times 240 \times 176 \mathrm{~mm}^{3}$ ). To get a ground truth and enable valid comparison, we considered the full FOV dataset $(R=1)$ that was downsampled a posteriori to simulate different sampling schemes and assess their performance.

\subsection{Reconstruction results: a comparative study}

We first compared SENSE reconstruction (no regularization) with the HYR ${ }^{2}$ PICS method on a convential pMRI undersampling scheme so as to emphasize the impact of hybrid regularization. As illustrated in Fig. 2, SENSE reconstruction shows strong and repeated artifacts along the phase encoding direction (y-axis) that are perfectly removed using the $\mathrm{HYR}^{2}$ PICS procedure. On this slice, our approach coupled with conventional pMRI undersampling also enhances the cerebellum and brain stem. However, on other slices incoherent sampling schemes may appear more powerful. This point is illustrated in Fig. 3 where 2D random points and checkerboard undersampling schemes provide more accurate reconstructions than any line-based undersampling alternative. Quantitative assessment of reconstruction quality through the computation of Signal-to-Noise Ratio (SNR) confirms our visual impressions.
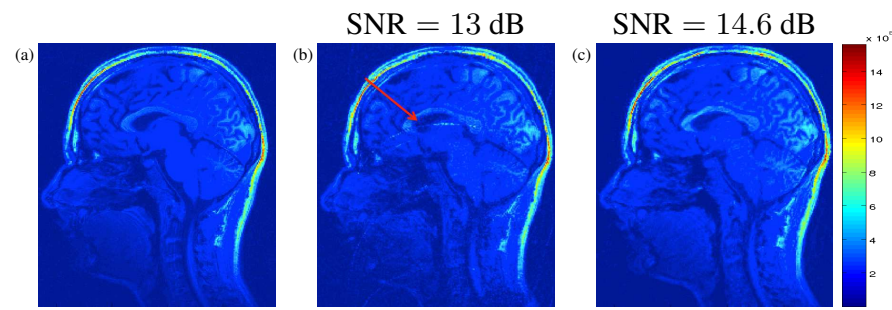

Fig. 2. Reconstruction results for $R=4$ (slice 82): (a): Reference image. (b):SENSE imaging. (c): ND random line undersampling.
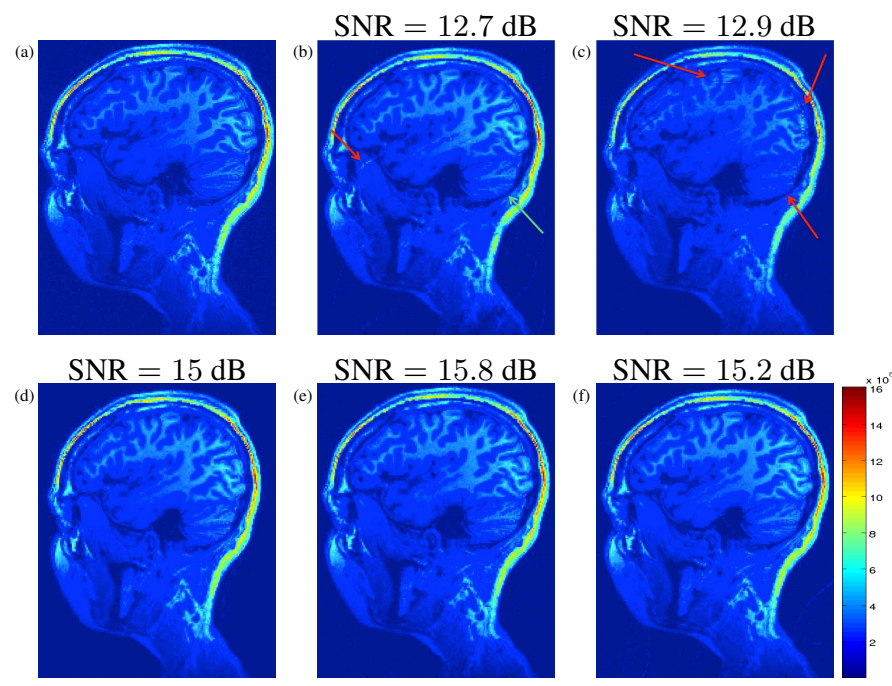

Fig. 3. Reconstruction results for $R=4$ (slice 42): (a): Reference image. (b): pMRI line undersampling. (c): ND random line undersampling. (d): Radial scheme with uniformly random angles. (e): 2D random points. (f): checkerboard scheme.

\section{CONCLUSION}

We proposed to unify CS and parallel imaging in a joint reconstruction framework that amounts to solving a large scale ill-posed inverse problem. Our HYR ${ }^{2}$ PICS approach therefore embodied hybrid penalization in the wavelet and image domains to regularize this problem. The ensuing convex but nonsmooth criterion was minimized by the CP primal-dual algorithm. We illustrated the impact of regularization and then demonstrated the interest of $k$-space undersampling schemes that depart from selecting one phase encoding line out of $R$ in parallel imaging. Interestingly, our reconstruction results appeared in agreement with the coherence assessment performed prior to the reconstruction. Future work will investigate other sparsifying transforms like tight frames.

\section{REFERENCES}

[1] K. P. Pruessmann, M. Weiger, M. B. Scheidegger, and P. Boesiger, "SENSE: sensitivity encoding for fast MRI," Magn Res in Med, vol. 42, no. 5, pp. 952-, 1999.

[2] D. K. Sodickson and W. J. Manning, "Simultaneous acquisition of spatial harmonics (SMASH): fast imaging with radiofrequency coil arrays," Magn Res in Med, vol. 38, no. 4, pp. 591-, 1997.

[3] M. A. Griswold, P. M. Jakob, R. M. Heidemann, M. Nittka, V. Jellus, J. Wang, B. Kiefer, and A. Haase, "Generalized autocalibrating partially parallel acquisitions GRAPPA," Magn Res in Med, vol. 47, no. 6, pp. 1202-, 2002.

[4] M. Lustig and J.M Pauly, "SPIRiT: Iterative self-consistent parallel imaging reconstruction from arbitrary k-space.," Magn Res in Med, vol. 64, no. 2, pp. 457-, 2010.

[5] E. Candès, J. Romberg, and T. Tao, "Robust uncertainty principles: exact signal reconstruction from highly incomplete frequency information," IEEE Trans. Inf. Theory, vol. 52, no. 2, pp. 489-, 2006.

[6] D. L. Donoho, M. Elad, and V. N. Temlyakov, "Stable recovery of sparse overcomplete representations in the presence of noise," IEEE Trans. Inf. Theory, vol. 52, pp. 1289-, 2006.

[7] M. Lustig, D.L Donoho, and J.M Pauly, "Sparse MRI: The application of compressed sensing for rapid MR imaging," Magnetic Res in Med, vol. 58, no. 6, pp. 1182-, 2007.

[8] K. T. Block, Ma. Uecker, and J. Frahm, "Undersampled radial MRI with multiple coils. Iterative image reconstruction using a total variation constraint," Magn Res in Med, vol. 56, no. 7, pp. 1086-, 2007.

[9] B. Liu, F.M. Sebert, Y. Zou, and L. Ying, "SparseSENSE: randomlysampled parallel imaging using compressed sensing," in Proc of the 16th ISMRM, Toronto, May 2008, p. 3154.

[10] D. Liang, B. Liu, J.-J Wang, and L. Ying, "Accelerating SENSE using compressed sensing," Magn Res in Med, vol. 62, no. 6, pp. 1574-, 2009.

[11] L. Chaari, J.-C. Pesquet, A. Benazza-Benyahia, and P. Ciuciu, "A wavelet-based regularized reconstruction algorithm for SENSE parallel MRI with applications to neuroimaging," Medical Image Analysis, vol. 15, no. 2, pp. 185-, 2011.

[12] L. Chaari, J.-C. Pesquet, Amel Benazza-Benyahia, and P. Ciuciu, "A hierarchical Bayesian model for frame representation," in 35th Proc. IEEE ICASSP, Dallas, TX, USA, 2010, pp. 4086-.

[13] Y Nesterov, "Gradient methods for minimizing composite objective function," ReCALL, vol. 76, 2007.

[14] P.L. Combettes and J.-C. Pesquet, "A proximal decomposition method for solving convex variational inverse problems," Inverse Problems, vol. 24, no. 6, pp. 27, 2008.

[15] A. Chambolle and T. Pock, "A first-order primal-dual algorithm for convex problems with applications to imaging," JMIV, 2011.

[16] X. Ye, Y. Chen, W. Lin, and F. Huang, "Fast mr image reconstruction for partially parallel imaging with arbitrary k-space trajectories," IEEE Trans. Med. Imag., vol. 30, no. 3, pp. 575-, 2011.

[17] P. L. Combettes and J.-C. Pesquet, "Primal-Dual Splitting Algorithm for Solving Inclusions with Mixtures of Composite, Lipschitzian, and Parallel-Sum Type Monotone Operators," Set-Val. and Var. Anal., 2011. 